\title{
Design of a Miniaturization Laser Triangulation Probe
}

\author{
Cao Miao ${ }^{1}$ and Yao Qinghua ${ }^{2}$ \\ 1 Changchun University of Science and Technology, Jilin Changchun, 130022, China \\ 2 Changchun University Of Technology, Jilin Changchun, 130012, China \\ 79816540@qq.com
}

\begin{abstract}
This paper puts forward a new design idea of single lens according to the principle of optical triangulation. The minitype laser triangle probe is designed on the basis of the common direct laser triangulation whose light path is improved. A beam splitter lens is introduced to the path, and the focusing lens and imaging lens are into one. The beam splitter lens, detector and focusing lens are coaxial, so the system is more compact, and the whole measurement system's volume is reduced. Optical system is simulated by optical design software ZEMAX (focus $=20 \mathrm{~mm}$, Pupil diameter $=4 \mathrm{~mm}$, and TOTR=20.5mm), so the whole system can achieve miniaturization. With appropriate laser and photoelectric detectors , larger scope of work and higher precision of measurement is obtain. The system adaptability to the measurement environment is improved, and this system can be widely used in the industrial online detection and military fields.
\end{abstract}

Keywords: Laser triangulation, Scheimpflug condition, Miniaturization, Beamsplitter, Triangulation probe

\section{Introduction}

The Laser Triangulation Probe is a kind of high precision and non-contact measurement instrument, which is based on the principle of laser triangulation measurement and widely used in industrial real-time online detection [1-4]. Compared with the traditional contact measuring method, the characteristics of laser triangulation non-contact measurement system are high-accuracy, fast speed, miniaturization and integration. According to the relations between the incident light and the measured surface normal, laser triangulation measurement can be divided into direct type structure and the oblique type [5-7]. These two kinds of triangulation ranging method all use the collimating-focusing lens and the imaging lens group. The two lens groups require certain distance and angle in the layout, and the layout must be in want of a certain space [8-10]. So the whole system is comparatively large, which will control of the system measurement environment. In comparison, the direct measurement method has the advantages of small volume, small imaging spot, light Intensity focused, stable imaging facula with the mobile object [11]. Therefore, the optical system was improved which is based on the direct type triangulation ranging method. In the guarantee of higher measurement accuracy, more compact structure, reducing the volume of the system, and that will enable the system to adapt to diverse environment measurement work.

\section{The Ranging Principle of Direct Laser Triangulation}

Direct laser triangulation is superior to oblique type in decreasing the volume of the instrument, so this design is on the straight type. The measuring light path is as shown 
in Figure 1. The laser beam emitted from the light source through the collimating lens is incident on the measured surface, forms diffuse reflection spot, imaging to the photosensitive detector surface through the Imaging lens. When the target position is changed, the position of the light spot is changed on the photoelectric detector. The change of micro position of object surface is calculated through the change position of the light spot detector.

According to the principle of geometrical optics, the image plane is only an accurate focus position, while the rest positions are in different degree of defocus state. This state can cause the image point dispersion, make system measurement not completely, and produce large errors. The traditional laser triangulation ranging method is larger volume, therefore the structure is considered appropriately to improve to realizing the miniaturization.

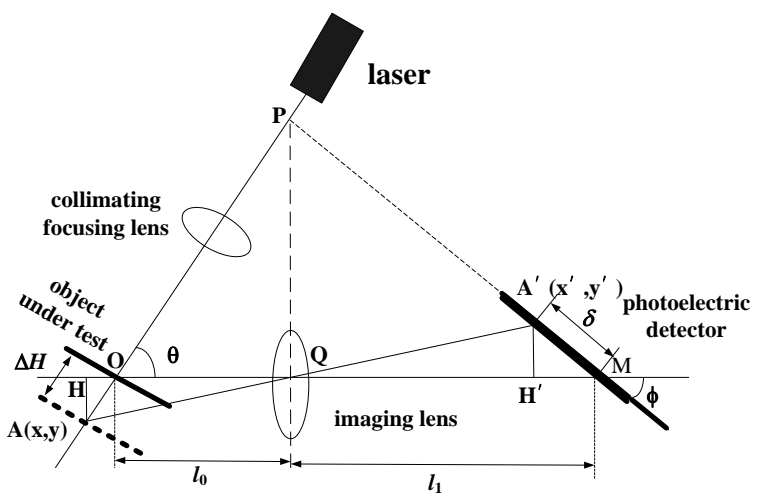

Figure 1. Direct Laser Triangulation Optical Path

\section{Improvement of Direct Laser Triangulation Ranging Principle}

\subsection{Improved Design Scheme}

By the research of direct laser triangulation ranging method, now some improvement is to the optical path. A beam splitter lens is introduced in the light path, and the focusing and imaging lenses are into one. The beam splitter lens, detector and condensing lens are coaxial in the space layout, which can make the structure more compact and small, and has a higher measurement environment adaptability and convenient to use. The light path is as shown in Figure 2.

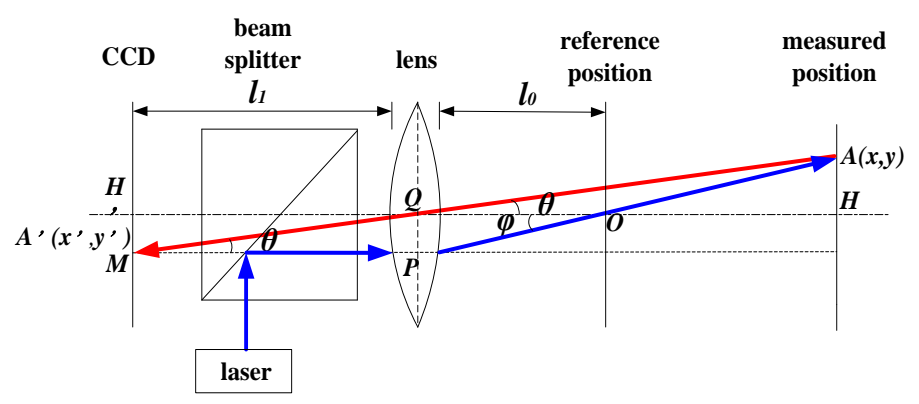

Figure 2. Optical Diagram of Laser Triangulation System with Single Lens 
The incident light passes through the beam splitter with $45^{\circ}$ angle and is reflected in a direction to condenser lens paralleled to the optical axis of the convex lens, which is rayed to the measured object with concentrated. Light (scattering light) reflected by the measured object is through the focus projection lens to the beam splitter. The beam splitter transmits part of the light, so CCD is able to capture and convert it into electrical signals. The signals with information of measured object are transmitted to the host computer for analysis and calculation by the data acquisition system, thus micro displacement measurement of the object is realized.

CCD position measurement principle is as shown in Figure 3. Waveform A is CCD measurement period and driving signal integral pulse, and waveform B is spot output signal of CCD. The signal $A$ and $B$ are act on the set and reset of the trigger respectively. The output waveform $\mathrm{C}$ is produced, and the time interval of the $\mathrm{C}$ signal expresses spot position on the CCD. Different measured distance correspond the different light spot position of CCD, which produces the different waveform $\mathrm{B}$ and different length $\mathrm{C}$ waveform. The time interval of the $\mathrm{C}$ waveform can be measured by the pulse number. The drive signal reset pulse is as a clock signal, which is logical multiplied by $\mathrm{C}$ signals. The position signal $\mathrm{D}$ indicated by pulse number is got. If the reset pulse is $1 \mathrm{MHz}, \mathrm{CCD}$ pixel intervals is $7 \mu \mathrm{m}$, optical magnification rate is $\beta$, the measurement pulse number is $\mathrm{N}$, the measured changes distance is :

$$
L=7 N / \beta
$$

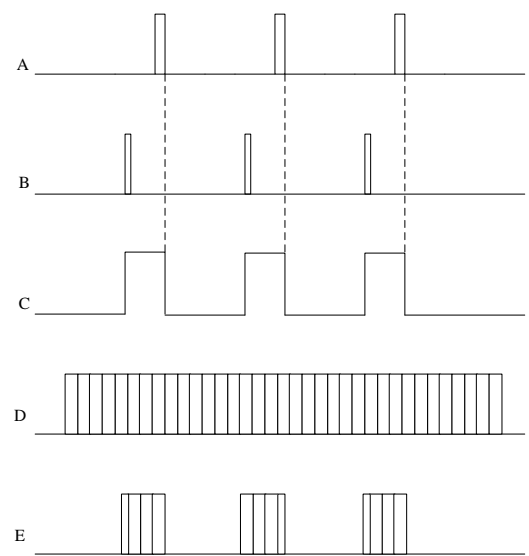

Figure 3. CCD Position Measurement Principle Diagram

\subsection{Improved Direct Type Scheimpflug Conditions}

In order to make the spot images of the detector surface clearly imaged, optical path layout needs to satisfy the Scheimpflug condition. This design selects the line array CCD as the detector, as shown in Figure 2. $l_{0}$ is the distance from the intersection of incident laser beam axis and the imaging lens axis to the imaging lens front main surface. $l_{l}$ is distance from the main imaging lens surface to the receiver center of CCD imaging. $f$ is imaging lens's focal length. The angle $\varphi$ is between the axis of incident laser beam and the imaging lens. The angle $\theta$ is between the axis of reflected laser beam with the imaging lens, and other parameters are shown in the Figure 2.

It is a reference measurement point with the spot, which is irradiated onto the surface of the measured on the optical axis of imaging lens. The coordinate system is established with $\mathrm{O}$ as the origin point of coordinates, and the optical axis of the lens is 
as the $\mathrm{X}$ axis. According to the principle of geometrical optics, the origin of the right is the "+", and "-" on the left. The spot of origin is imaged clearly through the imaging lens on the $\mathrm{CCD}$, according to the imaging conditions of paraxial, optical system to meet:

$$
\frac{1}{l_{0}}+\frac{1}{l_{1}}=\frac{1}{f}
$$

If the measured point moves small distances from the reference position along the direction optical axis, i.e., it moves to a point in the Figure 2, which is set $\mathrm{A}(\mathrm{x}, \mathrm{y}) . \mathrm{A}$ ' $\left(x^{\prime}, y^{\prime}\right)$ is the image point of A through the imaging lens. A (x, y) is the imaging point through imaging lens (for the following formula convenient, it is also named $\mathrm{M}$ ). In accordance with the paraxial imaging conditions, A spot formed by the imaging lens from A, images clearly meeting:

$$
\frac{1}{x-l_{0}}+\frac{1}{l_{0}-x^{\prime}}=\frac{1}{f}
$$

By the mathematical relationships, $\triangle A H Q \backsim \triangle A^{\prime} H^{\prime} Q$, the relation is available :

$$
\frac{\overline{A H}}{\overline{H Q}}=\frac{\overline{A^{\prime} H^{\prime}}}{\overline{H^{\prime} Q}}, \text { i.e., : }
$$

$$
\frac{y}{x-l_{0}}=\frac{y^{\prime}}{l_{0}-x^{\prime}}
$$

In the $\triangle \mathrm{AHQ}$, the geometric relation is:

$$
y=x \tan \theta
$$

The relationship is available from formula (2) - (5):

$$
y^{\prime}=\left(\frac{l_{0}-f}{f} \cdot \tan \theta\right) \cdot x^{\prime}-\frac{l_{0}^{2}}{f} \tan \theta
$$

A linear relationship is from the formula (6), and which is reflected that the object moves along the direction of incident light in the CCD receiver. A spot which is formed on the object surface by laser beam is imaged on the CCD whose trajectory is a straight line. In order to obtain image clearly, photoelectric receiver CCD should be placed in the form on this line track. According to the above coordinate system, the position relationship of the incident laser, the imaging lens and the CCD are determined by the geometry relationship.

In the $\triangle \mathrm{QPO}$

$$
\tan \phi=\frac{\overline{P Q}}{\overline{Q O}}
$$

In the $\triangle \mathrm{QPM}$

$$
\tan \theta=\frac{\overline{P Q}}{\overline{P M}}
$$

According to the (7) (8), (9) can be obtained

$$
\tan \varphi=\frac{\overline{P M}}{\overline{Q O}} \tan \theta=\frac{l_{1}}{l_{0}} \tan \theta
$$


The formula (9) is "Scheimpflug" conditions, which is must meet in the constant focus system. The imaging lens and the CCD can be determined by the position of laser from the formula (8).

Formula (10) is from (1) and (9):

$$
\tan \theta=\frac{l_{0}-f}{f} \tan \varphi
$$

The position of the CCD detector can be determined by (10). After the beam splitter is applied, the energy of light source needs to be split by the beam splitter twice. Although light beam splitter with $50 \%$ ratio is used in the design, that light signal energy captured by CCD reaches a maximum value. At this time the energy is only $25 \%$ of source, so the laser light source power and stability have higher requirements in this design. The semiconductor laser development, at present, is very mature. It is easy to buy laser above $10 \mathrm{mw}$, so this also greatly increase the feasibility of the scheme

\subsection{The Design Results}

Only one lens is applied, so it is restrictive in imaging quality. Therefore, the first lens surface employs even aspheric surface. The laser wavelength of system $\lambda=650 \mathrm{~nm}$, Focal length $f^{\prime}=20 \mathrm{~mm}$, Pupil diameter is $4 \mathrm{~mm}$, the total length of the system (TOTR)is $20.5 \mathrm{~mm}$, Even with the beam splitter lens, the whole system can meet the requirements of miniaturization.

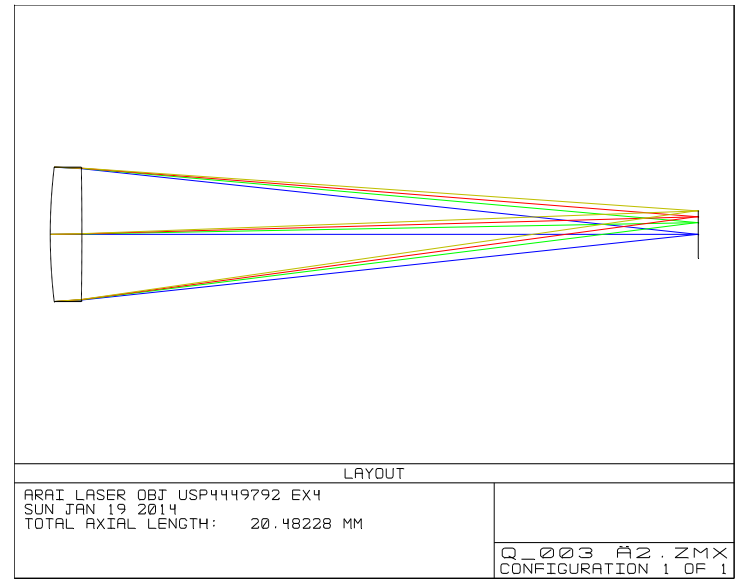

Figure 4. The Optical System Structure Diagram 


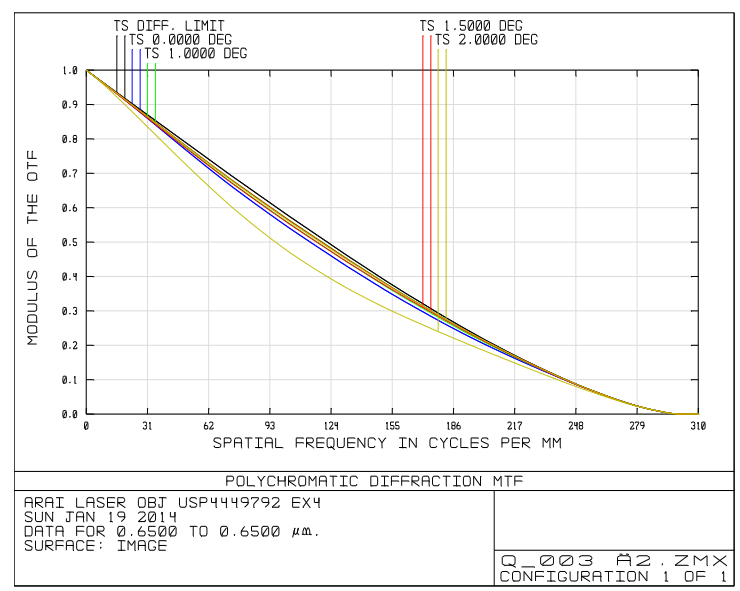

Figure 5. Modulation Transfer Function Curve

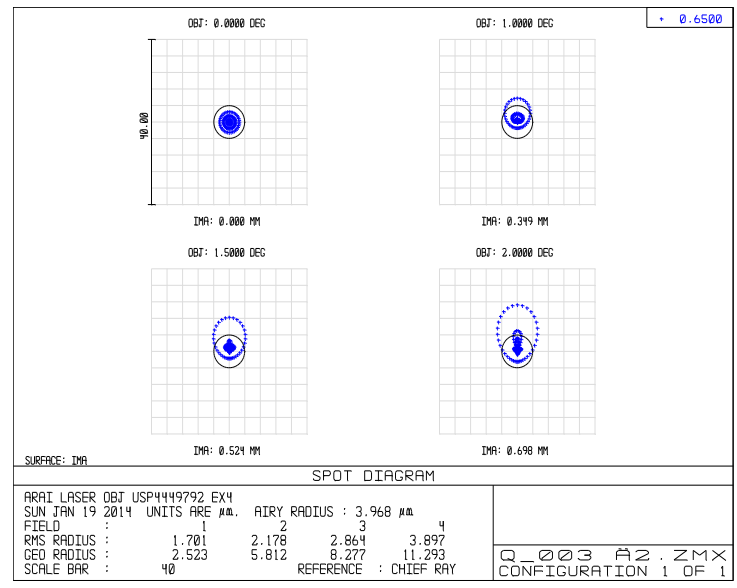

Figure 6. Spot Diagram

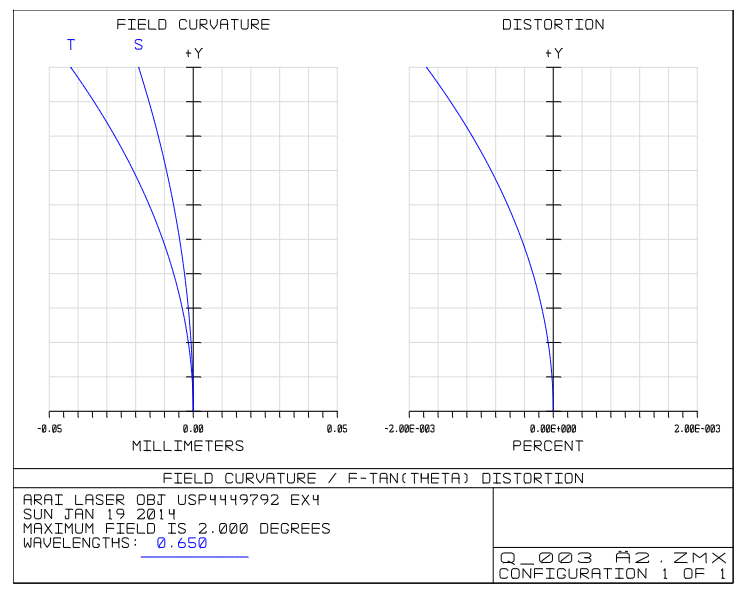

Figure 7. Astigmatism Fieldfield and Distortion Curve

The imaging quality is as shown in Figure 4-7. Transfer function (MTF) is close to the diffraction limit in the whole frequency range. Only the edge field of meridian transmission function curves is slightly exceeding, the root mean square radius in spot 
diagram is basically in the Airy pattern, and the relative distortion is less than $0.002 \%$. By choosing appropriate laser and photoelectric detector, and with the optical system, that can meet the high precision of laser triangulation probe.

\section{Conclusions}

The laser triangulation method is a classical non-contact measurement method. The laser triangulation method is more and more widely used in all walks of life, even in the military field. At present the domestic and foreign various research institutions are in high precision, high stability, high adaptability to the environment for the development of objectives for in-depth study of laser triangulation. The measuring accuracy is guaranteed in high precision in this paper for the purpose of reducing the measurement volume of the system. On the bases of the direct laser triangulation general optical system, by introducing the beam splitter and the reduction of a lens in the optical path, it adopts the layout of the normal coaxial. A single lens laser optical system is designed in triangulation measurement method with Zemax software, and the Scheimpflug conditions are derived in this structure. The design reduces the volume of the measurement system significantly, improves measuring environmental adaptability of the system, installs and uses more simply. After analysis, the performance of measurement with single lens optical system compared to the general direct laser triangulation, maintains a high measuring accuracy, and can get a larger working distance.

\section{Reference}

[1]. B. Perdan, D. Bracun, J. Diaci, et al., "Online assessment of power transmission belt geometry by using laser triangulation and profile parameterization”, Int J Adv Manuf Technol, vol. 49, (2010), pp. 177-184.

[2]. J. L. Vilaca, J. C. Fonseca and A. M. Pinho, "Non-contact 3D acquisition system based on stereo vision and laser triangulation", Machine Vision and Applications, vol. 21, no. 3, (2010), pp. 341-350.

[3]. A. Koulakezian, R. Ohannessian and H. Denkikian, "Wireless Sensor Node for Real-Time Thickness Measurement and Localization of Oil Spills", Proceedings of the 2008 IEEE/ASME international conference on advanced intelligent mechatronics, vol. 7, (2008), pp. 631-636.

[4]. S. Chang-ku, "Optical measurement technology", Tianjin: Tianjin university press, (2001).

[5]. P. Jin-ya, "Design of laser triangulation measurement system based on PSD", Wuhan: Huazhong university of science and technology, (2008).

[6]. G. Cai, J. Wu and Y. Xue, "Oil Spill Detection From Thermal Anomaly Using Aster Data in Yinggehai of Hainan, China", in Pro. of the Geoscience and Remote Sensing Symposium, (2007) July 23-28, pp. 898-900.

[7]. S. Ji-dong, Z. Wan-zhen and L. Bin, "A new optical angle measurement and compensation", Acta Photonica Sinica, vol. 40, no. 3, (2011), pp. 354-357.

[8]. L. J. Zeng, F. Yuan and D. Q. Sonu, "two-beam laser triangulations for measuring the position of a moving object", Optics and Lasers in Engineering, vol. 31, (1999), pp. 445-453.

[9]. H. Kevin and Q. Xiaoping, "Three Dimensional Dynamic Range Reduction Techniques", SPIE, vol. 5265, (2004), pp. 110-119.

[10].B. Drago, J. Matija and D. Janez, "Triangulation model taking into account light sheet curvature", Measurement Science and Technology, vol. 17, no. 8, (2006), pp. 2191-2196.

[11].J. J. Aguilar, J. Santolaria, D. Guillomia, et al., "Accuracy analysis of laser scanning probes used in coordinate measurement”, VDI Berichte, vol. 1860, (2004), pp. 739 744. 
International Journal of Control and Automation Vol. 8, No. 2 (2015) 